\title{
Initial Assessment of Hot-Melt Extrusion for Processing API with a Wide Range of Physical Properties to Form Consistent Solid Dispersion: HPMC-Affinisol Paracetamol System.
}

\section{T. Islam, J. Robertson, A. J. Florence}

University of Strathclyde

\section{Purpose}

The aim of this work is to:

1) Determine the viable processing window of HPMC-Affinisol polymer to use in hot melt extrusion.

2) Use this polymer for oral formulations with Paracetamol over a wide range of physical grades and determine the effect of API particle size on the nature of the resulting formulated mixtures.

3) Assess if HME can be used to produce a consistent output with wide ranges in physical input API properties.

\section{Methods}

HPMC (Affinisol ${ }^{\mathrm{TM}} 15 \mathrm{LV}$, Dow Chemical Co.) was fed into a Eurolab $16 \mathrm{~mm}$ co-rotating twin screw extruder at $1 \mathrm{~kg} / \mathrm{hr}$. A $3 \mathrm{~mm}$ die was used to produce the extruded strand. Three barrel temperatures $\left(150,180\right.$ and $\left.210^{\circ} \mathrm{C}\right)$ were used and the screw speed was adjusted between 100-1000rpm. Torque and pressure generated during extrusion were also monitored. The physical appearance of the extrudate was monitored for discolouration and clarity to determine the viability of the processing conditions for production of the extrudate. Three physical grades of 25\% (w/w) of Paracetamol (Mallinckrodt Chemical limited, D(v,0.5): micronized-10.3 $\mu$ m, powder-52.6 $\mu \mathrm{m}$ and granular-299 $\mu \mathrm{m}$ ) were used to produce solid dispersions of the Paracetamol in the polymer at the viable processing conditions determined during polymer extrusion. Differential scanning calorimetry (DSC) and powder X-ray diffraction (PXRD) analyses were carried out to assess whether the resulting formulations were amorphous. Physical mixtures of the drug-polymer were also analysed to provide a reference data set.

\section{Results}

The physical appearance of the extrudate of Affinisol assessed for discoloration and clarity across a range of processing temperatures and specific mechanical energy are shown (Figure 1). It was observed that Affinisol ${ }^{\mathrm{TM}}$ degraded/discoloured by a combined effect of thermal and mechanical energy input. From this screening test, a two level full factorial DOE was used to determine the final processing conditions $\left(180^{\circ} \mathrm{C}\right.$ barrel temperature, $250 \mathrm{rpm}$ screw speed at $1 \mathrm{~kg} / \mathrm{h}$ feed rate) for Affinisol ${ }^{\mathrm{TM}}$-Paracetamol formulations for the three grades of Paracetamol were extruded with Affinisol ${ }^{\mathrm{TM}}$ to yield a set of polymeric matrices. The DSC analysis (Figure 2) of the physical mixtures showed a slight shift of the glass transition temperature of Paracetamol with various grades whereas no melting endotherm of Paracetamol was observed for the extruded polymer-Paracetamol. These results indicate that the Paracetamol is in amorphous state within the extrudates. The PXRD data generated also showed lack of crystallinity of the polymer-drug mixtures and is in agreement with the DSC analysis. The extent amorphous material in the polymer-Paracetamol extrudates cannot be specified but it can be concluded that it is substantial.

\section{Conclusion}

1) Specific mechanical energy and shear stress produced due to various screw speed as well as processing temperature must be limited for successful extrusion of Affinisol ${ }^{\mathrm{TM}}$.

2) Thermal and PXRD data of the formulated extrudates indicated that a highly amorphous matrix was formed for a range of physical API grades.

3) HME could be a useful process for formation of amorphous solid forms independent of input API physical properties.
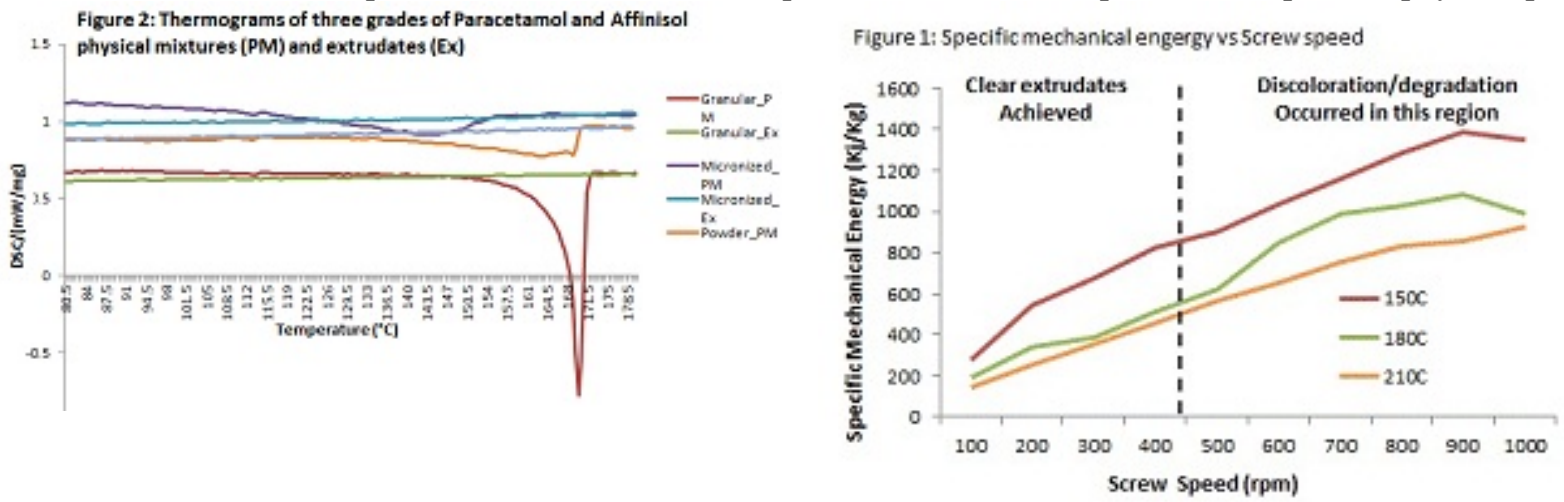